The thirteen chapters vary in length from just over one page to 45 pages. One chapter is devoted entirely to sarcoidosis with no explanation why this should be regarded as a blood disease. Pernicious anaemia, probably the most important association of blood and neurological disease, is inadequately described and the erroneous statement is reiterated that vitamin $B_{12}$ neuropathy occurs because vitamin $B_{12}$, but not folate, is involved in RNA synthesis. Possible effects of folate deficiency and folate therapy on epilepsy and psychological states are not discussed. The chapter on leukaemia fails to mention vincristine or effects of other antimetabolite drugs on the nervous system, with the exception of passing reference to methotrexate, nor are the different leukaemias or different neurological syndromes subdivided in any coherent fashion. Most of the chapters are anecdotal and contain no tables or summary and it is difficult for the reader to sort out the exact syndrome or the exact haematological disease under discussion.

\section{Principles of Electronics in Medical Research}

By D. W. Hill. Pp. 370, illustrated. Second edition. London: Butterworths, 1973. $£ 4.00$.

This is the second edition of the book which first appeared in 1965. During the intervening 7 years there have been some striking developments in the field of medical electronics, notably in the introduction of semiconductors and their consequent impact on digital and analogue integrated circuitry. This has resulted in increasing awareness of the potential applications of electronic devices in the practice of medicine in patient monitoring and resuscitation. The decreasing size and increasing reliability of electronic devices have resulted in their widespread application so that few hospital doctors can have escaped their impact.

The purpose of this book was, and remains, the provision of an introduction to the field for workers in medicine and biology. It is not intended as a working manual but rather as a means for the acquisition of the fundamentals of the subject for those who use and maintain this type of instrumentation in both ward and laboratory settings. Wisely, Dr Hill has given a descriptive rather than detailed approach to circuitry so that the reader will be provided with sufficient background to enable him to have meaningful contact with electronic engineers.

The book assumes little and the first few chapters provide a readable introduction to the principles of electricity and magnetism and the nature and properties of some of the devices continuously used in electronics, including power sources, meters, resistors, capacitors and thermistors. The $\mathrm{Q}$ chapters on d.c. and a.c. circuitry, semiconductors, amplifiers $\subseteq$ and oscillators are full and informative and there are $\overrightarrow{\bar{F}}$ numerous diagrams with a minimum of mathematics to $\stackrel{S}{\rightarrow}$ illustrate the principles described. Of particular interest to the physician and the biologist are sections dealing with $\frac{C}{0}$ patient safety, anaesthetic explosions and a useful chapter $\overline{\bar{\omega}}$ on digital techniques and analogue to digital converters.

There is no doubt that the book is well produced and $\varrho$ referenced and reasonably priced. It will amply meet the need, which is not uncommon, of the doctor or biologist who is suddenly confronted with the need to master the elements $\overrightarrow{0}$ of electronics. Its limitation is perhaps the deliberate exclusion of any practical advice concerning the construction or $\vec{\omega}$ maintenance of electronic circuits to meet particular needs or the sources of information that may be available to the? doctor with a particular need who lacks the services of an 3 electronic engineer. It will provide a valuable introduction to those who have developed an interest in this rewarding and occasionally frustrating field and excellent background $v$ material for those who would like to understand the devices of that they use.

\section{Renal Biopsy}

By D. B. Brewer. Pp. 103, illustrated. Second edition. $\stackrel{ }{\supset}$ London: Edward Arnold, 1973. £4.75.

The first edition of 'Renal Biopsy' was published in 1964. $\mathbb{D}$ The second edition shows relatively little change in spite of the passage of 9 years during which immunofluorescent $\frac{\mathbb{D}}{3}$ techniques and electron microscopy have made a number $\bar{g}$ contributions to renal biopsy interpretation. The chaptess on the normal kidney, nephrotic syndrome and glomeruknephritis have been re-written and there is a short technici 16 appendix on details of the method Professor Brewer uses \$ొట prepare $1 \mu \mathrm{m}$ sections.

Published work on quantitative assessment of biopsies is discussed.

These changes do not really warrant anyone with the first edition running out to replace it with the second. However, the merits of the book remain its excellent black and white $\frac{0}{8}$ photomicrographs, with the relevant clinical histories, and $a \varrho$ sound approach to diagnosis and classification based on the $\overrightarrow{\vec{O}}$ author's long experience in this field. 